\title{
OUTBREAK OF GASTROENTERITIS IN A RESIDENTIAL COLLEGE
}

Jeannine Liddle

formerly of Central Sydney Area Public Health Unit

Brett Campbell, Virginia Lawrence

Central Sydney Area Public Health Unit

$\mathrm{T}^{\mathrm{T}}$ his article describes the investigation of an outbreak of 40 cases of gastroenteritis at a residential college.

The Central Sydney Area Public Health Unit was contacted on June 17, 1996 with a report that several students from the college had presented to the same medical practice over the weekend (June 15-16) with gastroenteritis. Anecdotal evidence suggested it was usual for some students to report gastrointestinal symptoms at this time of year, when end-of-term examinations are held.

\section{METHODS}

As a matter of urgency, hygiene and infection control practices were reviewed with the college staff. Advice on how to minimise the spread of infection was given verbally both to staff and students. Information sheets describing the prevention and management of gastroenteritis were circulated to staff and displayed on student notice boards.

The kitchen area was inspected and found to be clean and well maintained. Methods of food preparation were reviewed with the chef and other kitchen staff, but no major problems were identified. Appropriate practices in food handling, storage and cooking, and general hygiene measures, were verbally reinforced. Samples of food prepared in the kitchens over the two weeks before the initial notification were not available for testing.

\section{Case definition}

The notifying doctor and the college's administrators were asked to compile a list of students who had complained of gastrointestinal symptoms over the weekend.

A convenience sample of cases was interviewed so a broad case definition could be developed. An initial questionnaire was distributed, both to refine the case definition and to identify further cases. The questionnaire covered demographic details (age, sex, occupation at the college, whether resident at the college), symptoms (onset, duration, severity, use of health services), contacts, and meals eaten outside the college. The questionnaire was circulated to all college staff and residents.

Stool specimens from some of the cases and from five of the kitchen staff were collected for examination and culture.

\section{Potential sources of infection}

Results from the first questionnaire implied a food origin for the disease. A second questionnaire, based on the menus from June $10-15$, was therefore given to a random sample of 40 students ( 20 cases and 20 controls) in an effort to determine the source of infection.

To discover whether food contamination was continuing, food samples were taken from the kitchens a week after the initial notification.

\section{RESULTS}

Fifteen suspected cases were identified by the notifying doctor and the college administration.

\section{Case definition}

All 20 staff completed the questionnaire, and 74 of 215 questionnaires were returned (a response rate of 34 per cent). After scrutiny of the responses, cases were defined as students or staff of the college presenting with diarrhoea (two or more runny stools

\section{The 1997 schools survey}

\section{$\checkmark$ Continued from page 35}

\section{PUTTING THE DATA TO WORK}

A professional development package will be prepared and disseminated by the end of 1997, drawing substantially on the survey's finding. It will use the health-promoting school concept ${ }^{1}$ as its framework and will address issues including support training for teachers, potential changes to school policy and practice, potential changes to the school environment, approaches to ensuring gender, socioeconomic and geographical equity, and approaches to engaging the support of parents, community organisations and other government sectors.

Looking further ahead, funding will be sought for controlled research to compare different approaches to promoting physical activity, physical skills and fitness among young people; and there is a possibility of developing the survey process as a monitoring tool, repeating it every three to five years.

Many lessons in developing collaborative research links were gleaned from the survey process, including the need to be responsive to shifts in administrative priorities and community sentiment about specific issues, and the need to be prepared to seize opportunities as they arise. We concluded that public health professionals should not underestimate the power of congenial personal relationships in these collaborations, and that researchers should take time to listen to, and understand, the goals and interests of potential partners and the expertise offered to help them meet those goals as well as their own.

1. NHMRC Health Advancement Standing Committee. Effective school health promotion: Towards health promoting schools. Canberra: Australian Government Publishing Service, 1996. 


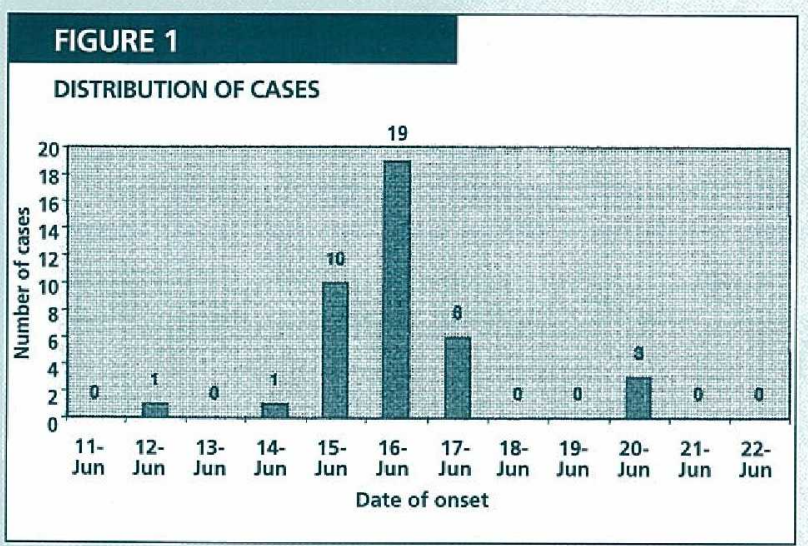

in 24 hours), or stomach ache and fever/chills, since June 10, 1996. Forty cases were identified from the questionnaire -37 students and 3 staff. Cases ranged in age from 17 to 45 years, with a median age of 19 years. Staff and students not affected were older (age range from 18 to 67 years, with a median age of 36 years). Symptoms lasted for up to nine days, with a median duration of three days. Many of the students (29) sought medical advice because they became ill at the time of their end-of-term examinations. No cases required hospital admission.

An epidemic curve (Figure 1) shows the distribution of cases. The narrow peak suggests a point source of foodborne disease, rather than one transmitted from person to person.

\section{The hypothesised cause - Campylobacter jejuni} Campylobacter jejuni was identified in stool specimens from three of the cases. This is a bacterium found in domestic animals, livestock and poultry. Infection usually occurs when food is inadequately cooked or is contaminated by other uncooked food. The incubation period is usually 2-10 days.

The stool specimens from the kitchen staff were negative for Campylobacter jejuni and for other possible causes of the epidemic. This indicated the important fact that staff in the public kitchens were not shedding the infective organism.

Twenty-one students replied to the second questionnaire ( 13 cases and 8 controls, giving an overall response rate of 53 per cent). No food or other specific source of Campylobacter jejuni was implicated in the results.

No Campylobacter species were identified in food samples taken after the initial notification, but faecal coliforms were identified in a sample of peanut butter. The peanut butter was in the self-service area, suggesting contamination by the students using the area.

\section{Follow-up}

The self-service area of the kitchen was closed on June 20. It was reopened after the holiday break with increased staff supervision.

After the investigation, the investigation team had a meeting with senior staff of the college to explain and discuss the results of the investigation and the control and prevention measures required. The Public Health Unit maintained contact with the college in the weeks after the last case to ensure prompt detection of any further cases.

The issue of hygiene, especially among students using the kitchen area, was highlighted when students returned from the holiday break. Additional notices emphasising hygiene (especially hand washing) were circulated to students and staff.

\section{Sequelae of the investigation}

This investigation strengthened relations between the PHU and the college and provided a basis for future work in preventing disease among the resident student population.

The PHU plans to review and update its questionnaires for investigating outbreaks of gastroenteritis and foodborne illnesses.

\section{PUBLIC HEALTH EDITORIAL STAFF}

The editor of the NSW Public Health Bulletin is Dr Michael Frommer, Director, Centre for Research and Development, NSW Health Department. Dr Lynne Madden is production manager.

The Bulletin aims to provide its readers with population health data and information to motivate effective public health action. Articles, news and comments should be 1,000 words or less in length and include a summary of the key points to be made in the first paragraph. References should be set out using the Vancouver style, the full text of which can be found in British Medical Journal 1988; 296:401-5.

Please submit items in hard copy and on diskette, preferably using WordPerfect, to the editor, NSW Public Health Bulletin, Locked Mail Bag 961, North Sydney 2059. Facsimile (02) 93919029.

Please contact your local Public Health Unit to obtain copies of the NSW Public Health Bulletin. The Bulletin can be accessed via the Internet from the NSW Health Department's World Wide Website, at http:/www.health.nsw.gov.au/public-health/phb/phb.html Back issues can be obtained from the Better Health Centre, Locked Mail Bag 961, North Sydney 2059.

Telephone: (02) 9954 1193, Facsimile (02) 99555196. 